\title{
THE FUNDAMENTAL GROUP AND GALOIS COVERINGS OF HEXAGONAL SYSTEMS IN 3-SPACE
}

\author{
J. A. DE LA PEÑA AND L. MENDOZA \\ Received 10 August 2005; Revised 1 August 2006; Accepted 11 October 2006
}

We consider hexagonal systems embedded into the 3 -dimensional space $\mathbb{R}^{3}$. We define the fundamental group $\pi_{1}(G)$ of such a system $G$ and show that in case $G$ is a finite hexagonal system with boundary, then $\pi_{1}(G)$ is a (non-Abelian) free group. In this case, the rank of $\pi_{1}(G)$ equals $m(G)-h(G)-n(G)+1$, where $n(G)$ (resp., $m(G), h(G)$ ) denotes the number of vertices (resp., edges, hexagons) in $G$.

Copyright @ 2006 Hindawi Publishing Corporation. All rights reserved.

\section{Introduction}

A hexagonal system is a finite-connected graph with all edges lying in regular hexagons. Hexagonal systems have been extensively studied, as the natural representations of hydrocarbon molecules, analyzing various physicochemical properties of the molecules represented. Spectral graph theory is often employed for this purpose because the algebraic invariants associated to the graphs contain relevant information about the molecular structure; see $[2,7]$. Additionally, the application of topological techniques has become increasingly important in the field over the past years; see $[4,6]$.

The consideration of benzenoid hydrocarbons as planar hexagonal systems is a welldeveloped theory [2, 7]. As first discussed by Wasserman [13], one may conceive of classes of hydrocarbons, including knotted rings and linked rings (catananes), with interesting topological properties. On this trend, in [12] it was reported the synthesis of the first molecular Möbius strip and in [3] the synthesis of a molecular trefoil knot. The development of nanotechnology makes relevant the consideration of nanotubes and other related hexagonal systems in 3-space; see [8].

In this work, we consider hexagonal systems, embedded into the 3-dimensional space $\mathbb{R}^{3}$. Given (a possibly infinite) hexagonal system $G$ stabilized by the action of a group $\Gamma$ which acts freely on the vertices of $G$, the natural quotient $\pi: G \rightarrow G / \Gamma$ is said to be a Galois covering defined by the action of $\Gamma$. For a finite hexagonal system $G$, we build a universal Galois covering $\pi: \widetilde{G} \rightarrow G$ (i.e., for any Galois covering $\pi^{\prime}: G^{\prime} \rightarrow G$, there is a unique morphism $\bar{\pi}: \widetilde{G} \rightarrow G^{\prime}$ with $\left.\pi=\pi^{\prime} \bar{\pi}\right)$. The map $\pi$ is defined by the action of the 
fundamental group $\pi_{1}(G)$. This group $\pi_{1}(G)$ is also the fundamental group of the $C W$ realization $c w(G)$ of the hexagonal system $G$.

We say that an edge $e$ in $G$ is on the boundary if $e$ belongs only to one hexagon in $G$. In case $G$ has no boundary and $c w(G)$ is orientable, a well-known argument implies that $c w(G)$ is a topological torus and therefore $\pi_{1}(G)=\mathbb{Z} \times \mathbb{Z}$ (see Proposition 2.1). For the case with boundary, we will prove the following result.

Theorem 1.1. Let $G$ be a finite hexagonal system with boundary, then $\pi_{1}(G)$ is a (nonAbelian) free group.

In the case considered in the above theorem, the rank of $\pi_{1}(G)$, denoted by $\operatorname{rk}(G)$, is an important invariant of the hexagonal system. The $\operatorname{rank} \operatorname{rk}(G)$ can be numerically obtained from the number of vertices $n(G)$, the number of edges $m(G)$, and the number of hexagons $h(G)$ of $G$, in the following way.

Theorem 1.2. Let $G$ be a finite hexagonal system with boundary. Then

$$
\operatorname{rk}(G)=m(G)-h(G)-n(G)+1 .
$$

The proof of Theorem 1.2 follows from some simplicial homology arguments and is given in Section 3. In Section 4, we consider the simply connected case, that is, $\operatorname{rk}(G)=0$ and show that $G$ is equivalent to a planar graph.

The results reported in this work will be used in forthcoming papers to describe the general structure of finite hexagonal systems with boundary and calculate its momenta, $\pi$-electron energy, and other important invariants.

The reader is referred to $[6,10]$ for an algebraic topology background and terminology not explained in the paper.

We denote by $\mathbb{Z}$ and $\mathbb{Q}$ the additive group of integers and the rational number field, respectively.

\section{Galois coverings and the fundamental group}

2.1. A hexagonal system $G=\left(G_{0}, G_{1}, \mathcal{H}(G)\right)$ is given by a set of vertices $G_{0}$, a set of edges $G_{1} \subset G_{0}^{2}$, and a set $\mathscr{H}(G) \subset G_{1}^{6}$ of hexagons, satisfying the following:

(H1) each edge $e=\{x, y\} \in G_{0}^{2}$ satisfies $x \neq y$ and belongs to a hexagon (i.e., there is $e^{\prime} \in G_{1}^{5}$ with $\left.\left(e, e^{\prime}\right) \in \mathscr{H}(G)\right)$;

(H2) each vertex $x \in G_{0}$ belongs to at most 3 edges in $G$ (i.e., the degree $d(x) \leq 3$ );

(H3) each hexagon $\left\{e_{1}, e_{2}, \ldots, e_{6}\right\}$ is formed by pairwise different edges with $e_{i} \cap e_{i+1}$ a single vertex, for $i=1, \ldots, 6$ and $e_{7}=e_{1}$. Moreover, two hexagons have at most one edge in common.

We write $\left\{e_{1}, e_{2}, \ldots, e_{6}\right\}$ for elements of $\mathcal{H}(G)$ and get the drawing in Figure 2.1. Observe that not necessarily for any sextuple $\left\{e_{1}, \ldots, e_{6}\right\} \in G_{1}^{6}$ with $e_{i}=\left\{x_{i}, x_{i+1}\right\} \in G_{0}^{2}$ and $x_{7}=x_{1}$, we get a hexagon in $G$.

2.2. Examples of hexagonal systems.

(1) Planar graphs associated to benzenoid hydrocarbons (see, e.g., Figure 2.2). 


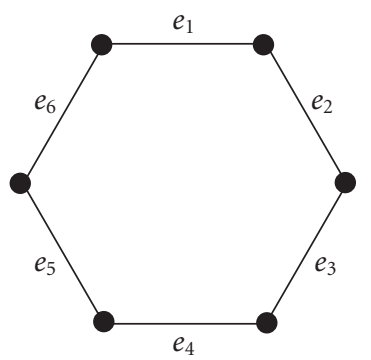

Figure 2.1<smiles>c1ccc2ccccc2c1</smiles>

(a)

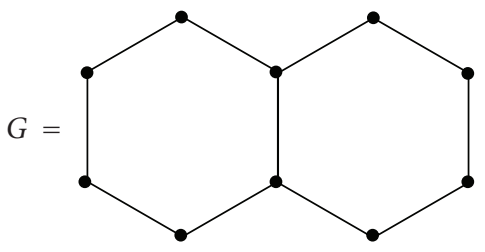

(b)

Figure 2.2

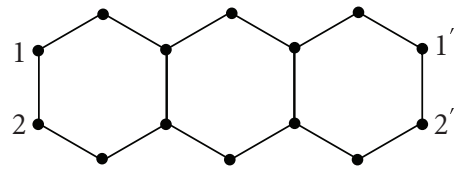

(a)

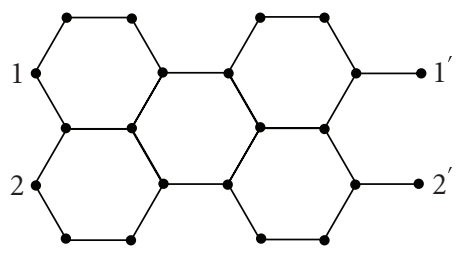

(b)

Figure 2.3

(2) Cylindrical tubes associated to nanotubes (identifying vertices with the same number). (See Figure 2.3.)

In (a), there are exactly 3 hexagons in $G$. The closed walks of length 6 forming the mouths of the cylinder are not hexagons in the system.

In (b), there are exactly 6 hexagons in $G$. Besides the 5 planar hexagons, there is an additional one formed by identifying $1 \leftrightarrow 1^{\prime}$ and $2 \leftrightarrow 2^{\prime}$.

(3) We will schematically draw cylinders as shown in Figure 2.4. In this case, the cylinder has $n$ half-twists. In case $n$ is odd, the cylinder is called a Möbius band.

(4) The torus is obtained by identifying the vertices and the edges indicated in Figure 2.5. All the 6-edge contours formed are hexagons in the torus. 


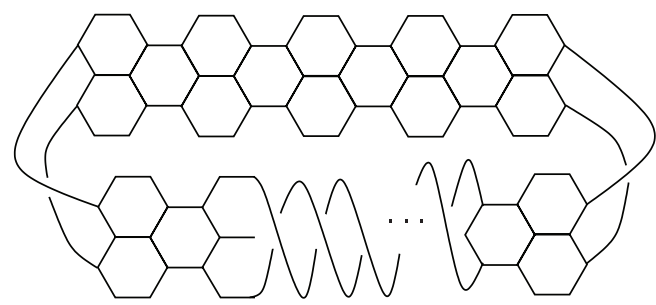

Figure 2.4

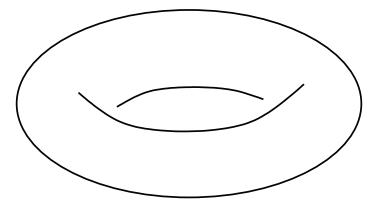

(a)

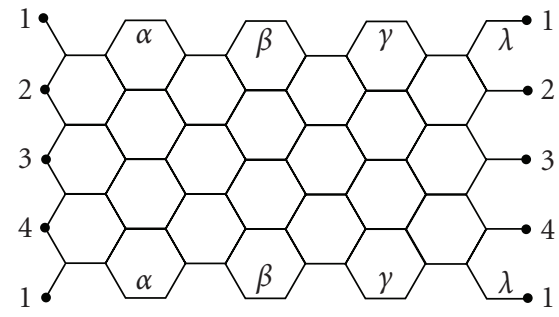

(b)

Figure 2.5

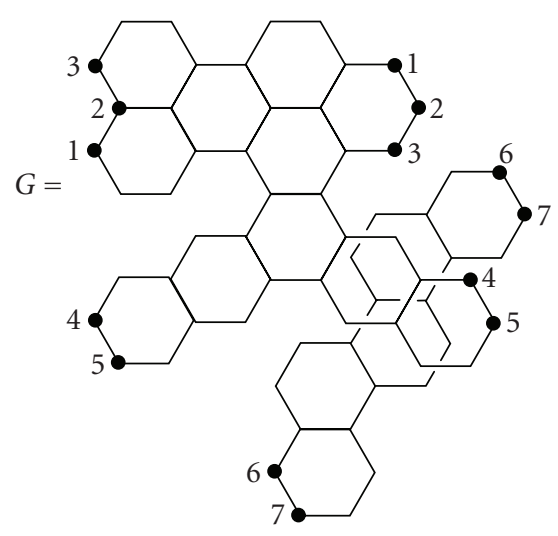

(a)

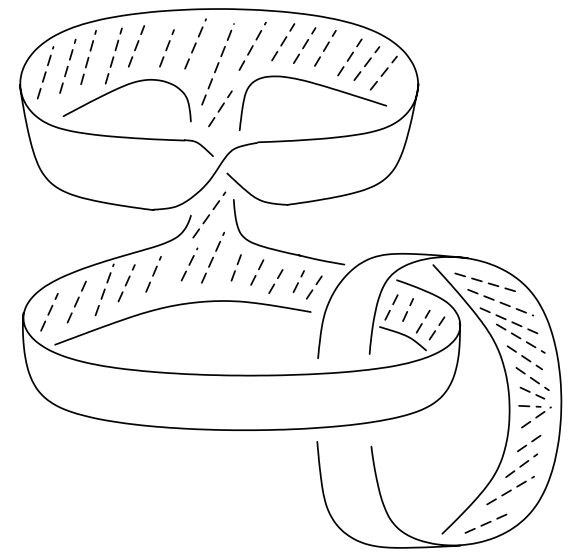

(b)

Figure 2.6

(5) The following hexagonal system is better understood by looking at the associated $C W$-complex shown in Figure 2.6.

2.3. Given a hexagonal system $G=\left(G_{0}, G_{1}, \mathcal{H}(G)\right)$, we consider an embedding $\xi: G \hookrightarrow \mathbb{R}^{3}$ where for each edge $e=\{x, y\}$, the image $\xi(e)$ is a differentiable curve and two edges $\xi(e)$ and $\xi\left(e^{\prime}\right)$ intersect only in the image $\xi(x)$ of a common vertex $x$ of $e$ and $e^{\prime}$. 
We recall that two homeomorphisms $f: \mathbb{R}^{3} \rightarrow \mathbb{R}^{3}$ and $g: \mathbb{R}^{3} \rightarrow \mathbb{R}^{3}$ are isotopic if there is a continuous function $H: \mathbb{R}^{3} \times[0,1] \rightarrow \mathbb{R}^{3}$ such that $H(x, 0)=f(x), H(x, 1)=g(x)$ for every $x \in \mathbb{R}^{3}$ and $H(x, t)$ is a homeomorphism for each fixed $t \in[0,1]$. It is known that either $f$ is isotopic to the identity or to a reflection function. In the first case, $f$ is said to be orientation preserving; in the second case, $f$ is said to be orientation reversing.

Two embeddings $\xi: G \hookrightarrow \mathbb{R}^{3}$ and $\eta: G \hookrightarrow \mathbb{R}^{3}$ are equivalent if there is an orientation preserving homeomorphism $f$ with $f \xi=\eta$. See [4] for a careful discussion.

The construction of a $C W$-complex $c w(G)$ associated to an embedding $\xi: G \hookrightarrow \mathbb{R}^{3}$ is fulfilled by fixing $\left\{\xi(x): x \in G_{0}\right\}$ as 0 -skeleton, $\left\{\xi(e): e \in G_{1}\right\}$ as 1-skeleton, and giving a set of differentiable functions $\left\{m_{h}:[0,1]^{2} \hookrightarrow \mathbb{R}^{3}: h \in \mathcal{H}(G)\right\}$ satisfying

$$
\partial\left(m_{h}\left([0,1]^{2}\right)\right)=m_{h}\left(\partial\left([0,1]^{2}\right)\right)=\xi(h),
$$

where the image of $m_{h}$ and $m_{h^{\prime}}$ may only intersect on $\xi(e)$ for an edge $e$ common to $h$ and $h^{\prime}$. Clearly, the homotopy class of $c w(G)$ depends only on the equivalence class of $\xi: G \hookrightarrow \mathbb{R}^{3}$.

We will say that the hexagonal system $G$ is orientable if the associated $C W$-complex $c w(G)$ is orientable, that is, there is a differentiable vector field $\Omega: c w(G) \rightarrow \mathbb{R}^{3} \backslash\{0\}$. It is well known that $G$ is orientable if and only if $c w(G)$ does not contain a Möbius band.

Proposition 2.1. Let $G$ be a hexagonal system without boundary and assume $G$ is orientable. Then $G$ is a torus.

Proof. Assume that $G$ has no boundary, then $d(x)=3$ for every $x \in G_{0}$. Then each edge belongs to 2 hexagons and each vertex to 3 hexagons, that is,

$$
h(G)+n(G)-m(G)=\frac{2}{3} m(G)+\frac{1}{3} m(G)-m(G)=0 .
$$

The compact orientable surface $c w(G)$ is homeomorphic to the sphere $(n=0)$ or to a torus with $n$ holes, $n \geq 1$. The Euler formula for the characteristic of $c w(G)$ yields

$$
2-2 n=\chi(c w(G))=n(G)-m(G)+h(G)=0 .
$$

Hence $n=1$ and $G$ is a torus.

2.4. Let $G$ be a connected hexagonal system. Fix $(s, t)$ an orientation of the edges of $G$, that is, for each $e=\{x, y\} \in G$, we have an arrow $s(e) \stackrel{e}{\longrightarrow} t(e)$, and the inverse arrow $t(e) \stackrel{e^{-1}}{\longrightarrow} s(e)$.

We will construct the fundamental group $\pi_{1}(G)$.

Fix a vertex $x_{0} \in G_{0}$. Consider the group $W\left(G, x_{0}\right)$ of all closed walks in $G$ starting and ending at $x_{0}$ (the trivial walk $\tau_{x_{0}}$ is the identity). Define a homotopy relation $\sim$ in $W\left(G, x_{0}\right)$ generated by the following.

(a) If $e=\{x, y\} \in G_{1}$, then $e e^{-1} \sim \tau_{t(e)}$ and $e^{-1} e \sim \tau_{s(e)}$. 
6 The fundamental group of hexagonal systems in 3-space

(b) If $\left\{e_{1}, \ldots, e_{6}\right\} \in \mathscr{H}(G)$, choose $\varepsilon_{i} \in\{1,-1\}$ such that $e_{1}^{\varepsilon_{1}} e_{2}^{\varepsilon_{2}} \cdots e_{6}^{\varepsilon_{6}}$ is an oriented path; then

$$
e_{i}^{\varepsilon_{i}} e_{i+1}^{\varepsilon_{i+1}} \cdots e_{6}^{\varepsilon_{6}} e_{1}^{\varepsilon_{1}} \cdots e_{i-1}^{\varepsilon_{i-1}} \sim \tau_{a_{i}}
$$

where $a_{i} \in\left\{s\left(e_{i}\right), t\left(e_{i}\right)\right\}$.

(c) If $u \sim v$, then $w u w^{\prime} \sim w v w^{\prime}$, whenever the products make sense.

We define $\pi_{1}\left(G, x_{0}\right)=W\left(G, x_{0}\right) / \sim$ the set of homotopy classes of closed walks at $x_{0}$. Clearly, $\pi_{1}\left(G, x_{0}\right)$ inherets the group structure of $W\left(G, x_{0}\right)$ and moreover, it does not depend on the chosen vertex $x_{0}$. We set $\pi_{1}(G)$ for $\pi_{1}\left(G, x_{0}\right)$.

The following is a more precise version of Theorem 1.1 stated at the introduction.

Theorem 2.2. Let $\xi: G \hookrightarrow \mathbb{R}^{3}$ be an embedding of the hexagonal system $G$. Consider the $C W$-complex $c w(G)$ and the fundamental group $\pi_{1}(G)$ associated to $G$. Then,

(a) $\pi_{1}(G)$ is the fundamental group of $c w(G)$,

(b) if $G$ has boundary, then $\pi_{1}(G)=\pi_{1}\left(\Delta_{G}\right)$ is the fundamental group of a graph $\Delta_{G}$, and therefore $\pi_{1}(G)$ is a (non-Abelian) free group.

Proof. (a) is clear from the definitions.

(b) A generalized hexagonal system $G=\left(G_{0}, G_{1}, \mathscr{H}(G)\right)$ satisfies $d(x) \leq 3$ for each $x \in$ $G_{0}$ and two hexagons in $\mathscr{H}(G)$ have at most one edge in common (i.e., $G$ satisfies conditions (H2) and (H3)). We will show, by induction on $h(G)$, that for a generalized hexagonal system $G$ with boundary, there exists a graph $\Delta_{G}$ such that $\pi_{1}(G)=\pi_{1}\left(\Delta_{G}\right)$ is a free group.

If $h(G)=0$, then $G$ is a graph and by classical theory, $\pi_{1}(G)$ is free of rank $m(G)$ $n(G)+1$.

Assume $h(G) \geq 1$. Since $G$ has boundary, we choose a hexagon $h \in \mathcal{H}(G)$ with edges $e_{i}=\left\{x_{i}, x_{i+1}\right\}$ for $1 \leq i \leq 6$ and $x_{7}=x_{1}$ such that $e_{1}, \ldots, e_{j}$ only belong to the hexagon $h$, and $d\left(x_{j+1}\right)=3$.

We consider the graph $G^{\prime}$ with vertices $G_{0} \backslash\left\{x_{2}, \ldots, x_{j}\right\}$, edges $G_{1}^{\prime}=G_{1} \backslash\left\{e_{1}, \ldots, e_{j}\right\}$, and $\mathscr{H}\left(G^{\prime}\right)=\mathscr{H}(G) \backslash\{h\}$. Then $G^{\prime}$ is a generalized hexagonal system with $h\left(G^{\prime}\right)<h(G)$. By induction hypothesis, $\pi_{1}\left(G^{\prime}\right)=\pi_{1}\left(\Delta_{G}\right)$ is a free group.

Choose $x_{0}$ a vertex in $G^{\prime}$. We will define a group homomorphism

$$
\rho: W\left(G, x_{0}\right) \longrightarrow W\left(G^{\prime}, x_{0}\right)
$$

for a fixed orientation $(s, t)$ of $G$ and the induced orientation in $G^{\prime}$. Indeed, let $w=$ $b_{1}^{\varepsilon_{1}} b_{2}^{\varepsilon_{2}} \cdots b_{m}^{\varepsilon_{m}} \in W\left(G, x_{0}\right)$ be a closed path at $x_{0}$, according to the orientation $(s, t)$. In case $b_{i} \neq e_{\ell}$ for every $1 \leq i \leq m$ and $1 \leq \ell \leq j$, then $\rho(w)=w \in W\left(G^{\prime}, x_{0}\right)$ is well defined.

Otherwise, we may assume $w=w^{3} w^{2} w^{1}$ with $w^{2}=e_{\ell}^{\alpha_{\ell}} \cdots e_{i}^{\alpha_{i}}$ for $1 \leq i<\ell \leq j$ and $w^{1}$, $w^{3}$ do not contain any arrow of the form $e_{k}^{\alpha_{k}}, 1 \leq k \leq j$. Then, $\rho(w)=w^{3} e_{\ell+1}^{-\alpha_{\ell+1}} \cdots e_{i-1}^{-\alpha_{i-1}} w^{1}$ $\in W\left(G^{\prime}, x_{0}\right)$ is well defined (see Figure 2.7).

It is straightforward to show that $w \sim w^{\prime}$ in $W\left(G, x_{0}\right)$ are sent by $\rho$ to homotopic paths in $W\left(G^{\prime}, x_{0}\right)$. Hence the induced function

$$
\bar{\rho}: \pi_{1}(G) \longrightarrow \pi_{1}\left(G^{\prime}\right)
$$




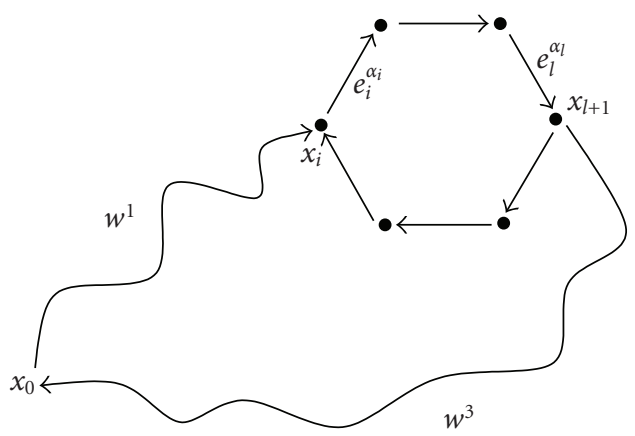

Figure 2.7

is a (surjective) group homomorphism. If $\bar{\rho}([w])=1$, where $[w]$ denotes the homotopy class of $w$, then there exists a sequence of homotopic cycles in $G^{\prime}$,

$$
\rho(w) \sim w_{1} \sim \cdots \sim w_{m}=\tau_{x_{0}} .
$$

It is enough to observe that $w \sim \rho(w)$ in $G$ and $\rho(w) \sim w_{1} \sim \cdots \sim w_{m}=\tau_{x_{0}}$ still holds in $G$. Hence $[w]=1$ and $\bar{\rho}$ is an isomorphism. This completes the proof of the theorem.

2.5. The proof of Theorem 1.1 provides a construction of the graph $\Delta_{G}$ such that $\pi_{1}(G)=$ $\pi_{1}\left(\Delta_{G}\right)$. Indeed, $\Delta_{G}$ is obtained from $G$ by deflating one by one the hexagons in $\mathscr{H}(G)$. We illustrate the procedure by an example.

Let $G$ be the hexagonal system obtained by identifying the vertices indicated with the same numbers as shown in Figure 2.8. Clearly, we may always consider an induced graph $\bar{\Delta}_{G}$ whose vertices are those $x$ in $\Delta_{G}$ with $d(x)=3$ such that $\pi_{1}\left(\Delta_{G}\right)=\pi_{1}\left(\bar{\Delta}_{G}\right)$.

2.6. We will prove that there is a hexagonal system $\widetilde{G}$ (which is infinite if $\pi_{1}(G) \neq 0$ ) stabilized by the action of the group $\pi_{1}(G)$ and such that the induced covering

$$
\pi: \widetilde{G} \longrightarrow \tilde{G} / \pi_{1}(G) \cong G
$$

is the universal Galois covering of $G$.

Fix a vertex $x_{0} \in G_{0}$ and an orientation $(s, t)$ of $G$, denote $\Pi=\pi_{1}\left(G, x_{0}\right)$, and for each vertex $x \in G_{0}$, fix a path $\gamma_{x}$ from $x_{0}$ to $x$, according to the orientation $(s, t)$. We define the hexagonal system $\widetilde{G}$ and the map $\pi: \widetilde{G} \rightarrow G$ as follows:

(i) vertices of $\widetilde{G}$ : set $\widetilde{G}_{0}=G_{0} \times \Pi$;

(ii) edges of $\tilde{G}$ : for $x \stackrel{\alpha}{\longrightarrow} y$ in $G_{1}$ and $[w] \in \Pi$, then $(x,[w]) \stackrel{\alpha}{\longrightarrow}\left(y,\left[\gamma_{y}^{-1} \alpha \gamma_{x} w\right]\right)$ in $\widetilde{G}_{1}$;

(iii) $\pi: \widetilde{G} \rightarrow G$ is defined as the natural projection;

(iv) hexagons in $\widetilde{G}$ : any cycle $\left\{\beta_{1}, \ldots, \beta_{6}\right\}$ such that $\left\{\pi\left(\beta_{1}\right), \ldots, \pi\left(\beta_{6}\right)\right\} \in \mathscr{H}(G)$. 
8 The fundamental group of hexagonal systems in 3-space

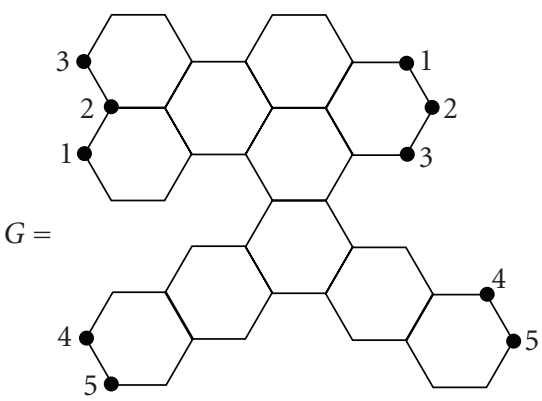

(a)

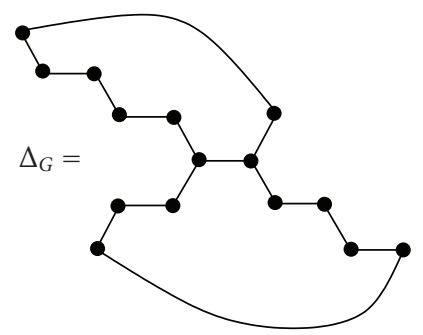

(c)

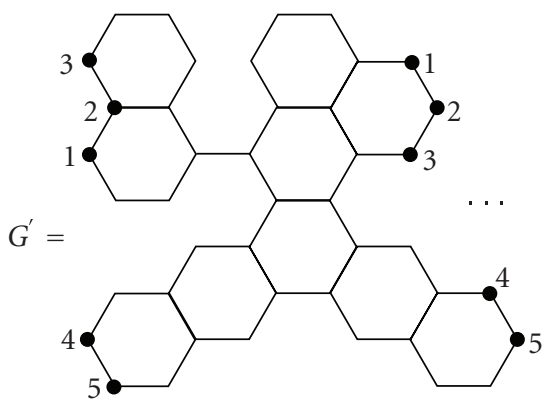

(b)

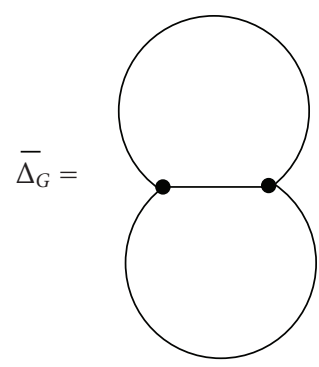

(d)

Figure 2.8

The group $\Pi$ acts on $\widetilde{G}$ and freely on the vertices of $\widetilde{G}$ (i.e., if $p \in \Pi$ and $p(y)=y$ for some $y \in \widetilde{G}_{0}$, then $\left.p=1\right)$. Indeed, $p=[v] \in \Pi$ and $(x,[w]) \in \widetilde{G}_{0}$, then $p(x,[w])=$ $(x,[v w])$; if $p(x,[w])=(x,[w])$, then $[v]=1$. Moreover, $\tilde{G} / \Pi=G$ as hexagonal systems.

Given any other Galois covering $\pi^{\prime}: G^{\prime} \rightarrow G$ defined by the action of a group $\Gamma$, we define a map $\bar{\pi}: \tilde{G} \rightarrow G^{\prime}$ of hexagonal systems. Indeed, consider an orientation $\left(s^{\prime}, t^{\prime}\right)$ of $G^{\prime}$ such that $\pi^{\prime}\left(s^{\prime}\left(e^{\prime}\right) \rightarrow t^{\prime}\left(e^{\prime}\right)\right)=s\left(\pi^{\prime}\left(e^{\prime}\right)\right) \rightarrow t\left(\pi^{\prime}\left(e^{\prime}\right)\right)$ and fix any $x_{0}^{\prime} \in G_{0}^{\prime}$ with $\pi^{\prime}\left(x_{0}^{\prime}\right)=$ $x_{0}$ and define $\bar{\pi}\left(x_{0},[1]\right)=x_{0}^{\prime}$. For any $x \in G_{0}$ and $[w] \in \Pi$, choose any path $\gamma_{x}$ from $x_{0}$ to $x$ in $G$, according to the orientation $(s, t)$ of $G$. The path $\gamma_{x} w$ goes from $x_{0}$ to $x$ and may be lifted to a path $\gamma_{x}^{\prime}$ in $G^{\prime}$, that is, $\pi^{\prime}\left(\gamma_{x}^{\prime}\right)=\gamma_{x} w$. If $x^{\prime}$ is the ending vertex of $\gamma_{x}^{\prime}$, we set $\bar{\pi}(x,[w])=x^{\prime}$. It is a straightforward exercise to see that this determines the wanted map $\bar{\pi}=\widetilde{G} \rightarrow G^{\prime}$. Additional properties of this map are stated in the following proposition.

Proposition 2.3. Let $G$ be a finite connected hexagonal system with boundary. Consider $\pi: \widetilde{G} \rightarrow G$ the Galois covering defined by the action of the fundamental group $\pi_{1}(G)$ as above. Let $\pi^{\prime}: G^{\prime} \rightarrow G$ be any Galois covering of hexagonal systems defined by the action of a group $\Gamma$, then the following holds:

(a) there is a map $\bar{\pi}: \widetilde{G} \rightarrow G^{\prime}$ of hexagonal systems such that $\pi=\pi^{\prime} \bar{\pi}$;

(b) the map $\bar{\pi}$ is a Galois covering defined by the action of a free group $H$ which is a normal subgroup of $\pi_{1}(G)$ such that $\pi_{1}(G) / H \stackrel{\sim}{\longrightarrow} \Gamma$. 


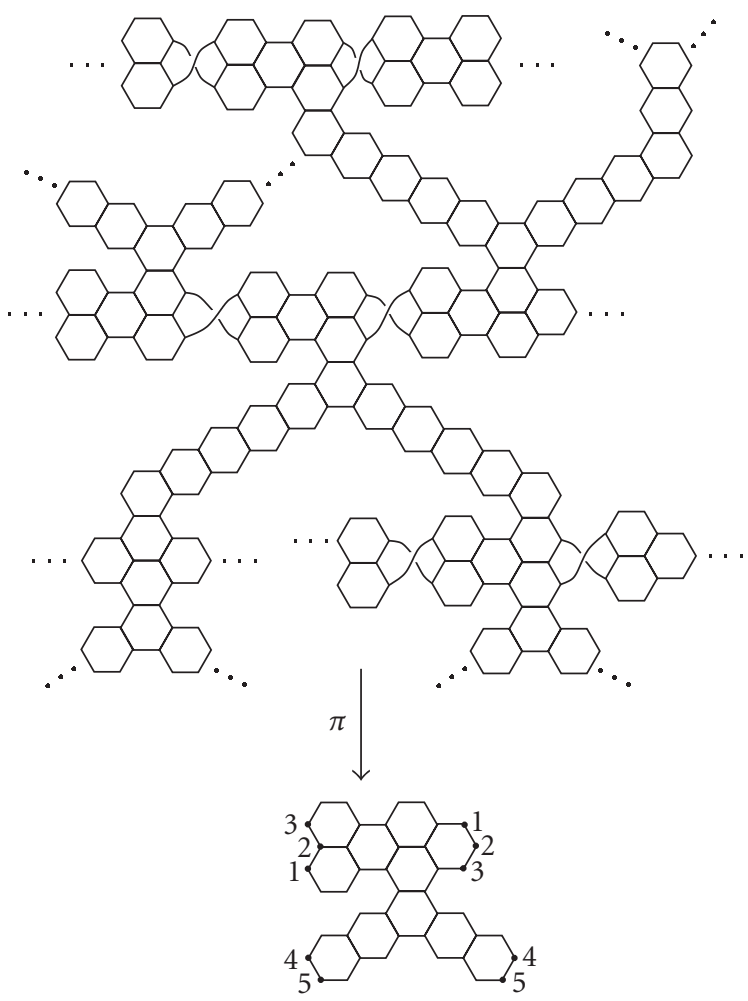

Figure 2.9

Remark 2.4. The construction of Galois coverings and the fundamental group of a hexagonal system follows similar arguments to those developed for finite dimensional algebras given as quotients of quiver algebras, see $[1,5,9,11]$. In particular, the proof of Theorem 1.1 is inspired in [11] where the concept of coverings in representation theory was introduced; the construction of the universal Galois covering follows [9].

2.7. As an example of the construction of the universal covering $\widetilde{G} \rightarrow G$, we will consider again the hexagonal system in Figure 2.8. We expect the following picture is selfcontained (see Figure 2.9).

Clearly, it is possible to define an embedding $\tilde{\xi}: \widetilde{G} \hookrightarrow \mathbb{R}^{3}$ and a $C W$-complex $c w(\widetilde{G})$ stable under the action of $\pi_{1}(G)$ and such $c w(\widetilde{G}) / \pi_{1}(G) \stackrel{\sim}{\longrightarrow} c w(G)$. We will not pursue this point.

\section{Cohomology and the rank of the fundamental group}

3.1. Let $G=\left(G_{0}, G_{1}, \mathscr{H}(G)\right)$ be a finite hexagonal system. Fix $(s, t)$ an orientation of $G$ and let $Z$ be an arbitrary Abelian (additive) group. Consider $C^{0}(G, Z)$ the set of $Z$-valued functions of $G_{0}$ and $Z^{1}(G, Z)$ the set of $Z$-valued functions on $G_{1}$ such that $\sum_{i=1}^{6} \varepsilon_{i} f\left(e_{i}\right)=$ 0 for every (oriented) hexagon in $\mathscr{H}(G)$ (see Figure 3.1). 


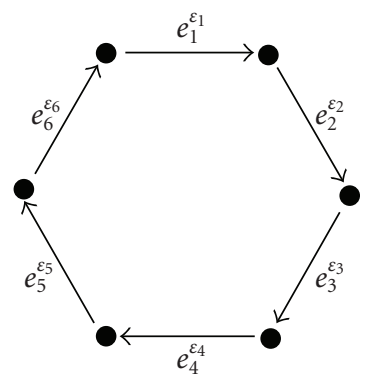

Figure 3.1

Proposition 3.1. Let $G$ be a finite connected hexagonal system with a fixed orientation $(s, t)$ of $G$. Let $Z$ be an arbitrary Abelian group. Then there is an exact sequence of Abelian groups:

$$
0 \longrightarrow Z \stackrel{d^{0}}{\longrightarrow} C^{0}(G, Z) \stackrel{d^{1}}{\longrightarrow} Z^{1}(G, Z) \stackrel{p}{\longrightarrow} \operatorname{Hom}\left(\pi_{1}(G), Z\right) \longrightarrow 0
$$

where

(1) $d^{0}(\lambda)=f_{\lambda}: G_{0} \rightarrow Z, x \mapsto \lambda$,

(2) $d^{1}(f): G_{1} \rightarrow Z, \alpha \mapsto f(t(a))-f(s(a))$,

(3) $g \in Z^{1}(G, Z)$, then $p(g): \pi_{1}(G) \rightarrow Z,\left[a_{1}^{\alpha_{1}} \cdots a_{s}^{\alpha_{s}}\right] \mapsto \sum_{i=1}^{s} \alpha_{i} g\left(a_{i}\right)$.

Proof. First we check that the maps are well defined.

For an (oriented) hexagon in $\mathscr{H}(G)$ as above and $f \in C^{0}(G, Z)$, we have

$$
\sum_{i=1}^{6} \varepsilon_{i} d^{1}(f)\left(e_{i}\right)=\sum_{i=1}^{6} \varepsilon_{i}\left[f\left(t\left(e_{i}\right)\right)-f\left(s\left(e_{i}\right)\right)\right]=0,
$$

and $d^{1}(f) \in Z^{1}(G, Z)$. If $g \in Z^{1}(G, Z)$, it is clear that $W\left(G, x_{0}\right) \rightarrow Z, a_{1}^{\alpha_{1}} \cdots a_{s}^{\alpha_{s}} \mapsto$ $\sum_{i=1}^{s} \alpha_{i} g\left(a_{i}\right)$ is well defined in homotopy classes and therefore, $p(g) \in \operatorname{Hom}\left(\pi_{1}(G), Z\right)$.

The composition $d^{1} d^{0}=0$ and for $f \in C^{0}(G, Z)$ and $w=a_{1}^{\alpha_{1}} \cdots a_{s}^{\alpha_{s}} \in W\left(G, x_{0}\right)$, we have

$$
p\left(d^{1}(f)\right)([w])=\sum_{i=1}^{s} \alpha_{i} d^{1}(f)\left(a_{i}\right)=\sum_{i=1}^{s} \alpha_{i}\left[f\left(t\left(a_{i}\right)\right)-f\left(s\left(a_{i}\right)\right)\right]=0 .
$$

Clearly, $d^{0}$ is injective. We check the remaining facts for the exactness of the sequence.

$\operatorname{Ker} d^{1} \subset \operatorname{Im} d^{0}$ : let $f \in C^{0}(G, Z)$ be such that $d^{1}(f)=0$. Assume we have a full connected subgraph $G^{\prime}$ of $G$ such that $f$ is constant in $G^{\prime}$. Take an edge $x \stackrel{a}{-} y$ such that $x \in G_{0}^{\prime}$ and $s(a)=x$, then $0=d^{1}(f)(a)=f(t(a))-f(s(a))=f(y)-f(x)$. Hence $f$ is constant in $G$.

$\operatorname{Ker} p \subset \operatorname{Im} d^{1}$ : let $g \in Z^{1}(G, Z)$ be such that $p(g)=0$. Fix a vertex $x_{0} \in G$ such that $\pi_{1}(G)=\pi_{1}\left(G, x_{0}\right)$ and for each vertex $x \in G_{0}$, choose a path $\gamma_{x}=a_{x_{1}}^{\alpha_{1}^{x}} \cdots a_{x m_{x}}^{\alpha_{m_{x}}^{x}}$ from $x_{0}$ to $x$ oriented according to the orientation $(s, t)$ of $G$. Define $f \in C^{0}(G, Z)$ by $f(x)=$ $\sum_{i=1}^{m_{x}} \alpha_{i}^{x} g\left(a_{x_{i}}\right)$. Then for $x \stackrel{a}{ } y$, say with $s(a)=x$, we get $a \gamma_{x}$ from $x_{0}$ to $y$. Since 
$p(g)=0$, then

$$
\begin{aligned}
d^{1}(f)(a) & =f(y)-f(x)=\sum_{i=1}^{m_{y}} \alpha_{i}^{y} g\left(a_{y_{i}}\right)-\sum_{i=1}^{m_{x}} \alpha_{i}^{x} g\left(a_{x_{i}}\right) \\
& =\left[g(a)+\sum_{i=1}^{m_{x}} \alpha_{i}^{x} g\left(a_{x_{i}}\right)\right]-\sum_{i=1}^{m_{x}} \alpha_{i}^{x} g\left(a_{x_{i}}\right)=g(a) .
\end{aligned}
$$

The map $p$ is onto: we define a function $r: \operatorname{Hom}\left(\pi_{1}(G), Z\right) \rightarrow Z^{1}(G, Z)$ by

$$
r(\varphi)(a)=\varphi\left(\left[\gamma_{t(a)}^{-1} a \gamma_{s(a)}\right]\right) .
$$

With arguments similar to the above, $r$ is well defined and $p \circ r$ is the identity in $\operatorname{Hom}\left(\pi_{1}(G), Z\right)$. This completes the proof of the statement.

3.2. The sequence (3.1) provides useful relations among the invariants of a hexagonal system. For instance, if $G$ is a torus, then $\pi_{1}(G)=\mathbb{Z} \times \mathbb{Z}$ and choosing $Z=\mathbb{Q}$ in (2.3) we get

$$
2=\operatorname{rk}(G)=\operatorname{dim}_{\mathbb{Q}} Z^{1}(G, \mathbb{Q})-n(G)+1 .
$$

It is not hard to show that

$$
\operatorname{dim}_{\mathbb{Q}} Z^{1}(G, \mathbb{Q})=m(G)-h(G)+1 .
$$

We get the formula

$$
0=m(G)-h(G)-n(G)
$$

that we encountered already in (2.3).

3.3. Proof of Theorem 1.2. Let $G=\left(G_{0}, G_{1}, \mathcal{H}(G)\right)$ be a finite hexagonal system with boundary and consider the exact sequence (3.1) for $Z=\mathbb{Q}$. We will prove that

$$
\operatorname{dim}_{\mathbb{Q}} Z^{1}(G, \mathbb{Q})=m(G)-h(G) .
$$

As in the proof of Theorem 1.1, we consider generalized hexagonal systems $G$ and we prove by induction on the number of hexagons $h(G)$ that $\operatorname{dim}_{\mathbb{Q}} Z^{1}(G, \mathbb{Q})=m(G)-h(G)$. Fix an orientation $(s, t)$ of $G$.

For $h(G)=0$, we get $\mathscr{H}(G)=\varnothing$ and $G$ is a graph with $Z^{1}(G, \mathbb{Q})=\mathbb{Q}^{G_{1}}$. Hence $\operatorname{dim}_{\mathbb{Q}} Z^{1}(G, \mathbb{Q})=m(G)$ in this case.

Assume $h(G) \geq 1$ and choose as in (2.4), a hexagon $h$ with edges $e_{1}, \ldots, e_{j}$ in the boundary. Suppose $x_{i} \stackrel{e_{i}^{\varepsilon_{i}}}{\longrightarrow} x_{i+1}$ for $1 \leq i \leq 6$ and $x_{7}=x_{1}$, and define $G^{\prime}$ the generalized hexagonal system with $G_{0}^{\prime}=G_{0} \backslash\left\{x_{2}, \ldots, x_{j}\right\}, G_{1}^{\prime}=G_{1} \backslash\left\{e_{1}, \ldots, e_{j}\right\}$, and $\mathscr{H}\left(G^{\prime}\right)=\mathscr{H}(G) \backslash\{h\}$. By induction hypothesis, we get

$$
\operatorname{dim}_{\mathbb{Q}} Z^{1}\left(G^{\prime}, \mathbb{Q}\right)=m\left(G^{\prime}\right)-h\left(G^{\prime}\right)=m(G)-h(G)-j+1 .
$$


12 The fundamental group of hexagonal systems in 3-space

We will define an exact sequence

$$
0 \longrightarrow\left\{v \in \mathbb{Q}^{j}: \sum_{i=1}^{j} \varepsilon_{i} v(i)=0\right\} \stackrel{\sigma}{\longrightarrow} Z^{1}(G, \mathbb{Q}) \stackrel{\rho}{\longrightarrow} Z^{1}\left(G^{\prime}, \mathbb{Q}\right) \longrightarrow 0
$$

with

(1) $\sigma(v): G_{1} \rightarrow \mathbb{Q}$,

$$
a \longmapsto \begin{cases}v(i) & \text { if } a=e_{i}(1 \leq i \leq j) \\ 0 & \text { else, }\end{cases}
$$

(2) $\rho(f): G_{1}^{\prime} \rightarrow \mathbb{Q}$, the restriction of $f: G_{1} \rightarrow \mathbb{Q}$.

Clearly, $\sigma$ and $\rho$ are well-defined homomorphisms with $\sigma$ mono and $\rho \sigma=0$.

$\operatorname{Ker} \rho=\operatorname{Im} \sigma:$ if $f \in Z^{1}(G, \mathbb{Q})$ with $\rho(f)=0$, then

$$
\sum_{i=1}^{j} \varepsilon_{i} f\left(e_{i}\right)=\sum_{i=1}^{6} \varepsilon_{i} f\left(e_{i}\right)=0, \sigma\left(f\left(e_{i}\right)_{i=1, \ldots, j}\right)=f .
$$

$\rho$ epi: let $f^{\prime} \in Z^{1}\left(G^{\prime}, \mathbb{Q}\right)$ and consider $f: G_{1} \rightarrow \mathbb{Q}$ with $\rho(f)=f^{\prime}$ extended by

$$
\varepsilon_{1} f\left(e_{1}\right)=-\sum_{i=j+1}^{6} \varepsilon_{i} f^{\prime}\left(e_{i}\right), \quad f\left(e_{i}\right)=0 \text { for } 2 \leq i \leq j .
$$

From this sequence we get the equality

$$
\operatorname{dim}_{\mathbb{Q}} Z^{1}(G, \mathbb{Q})=\operatorname{dim}_{\mathbb{Q}} Z^{1}\left(G^{\prime}, \mathbb{Q}\right)+j-1 .
$$

\section{Simply connected hexagonal systems}

4.1. We say that a hexagonal system $G$ is simply connected if $\pi_{1}(G)=0$.

Corollary 4.1. Let $G$ be a finite connected hexagonal system with boundary. Then $G$ is simply connected if and only if

$$
n(G)+h(G)-m(G)=1 .
$$

4.2. Let $G$ be a finite hexagonal system and $\xi: G \hookrightarrow \mathbb{R}^{3}$ be an embedding. Consider a projection $\pi: \mathbb{R}^{3} \rightarrow \mathbb{R}^{2}$ such that $\pi \xi: G \rightarrow \mathbb{R}^{2}$ satisfies

(1) $\left|(\pi \xi)^{-1}(\lambda)\right| \leq 2$ for each $\lambda \in \mathbb{R}^{2}$,

(2) $\left\{\lambda \in \mathbb{R}^{2}:\left|(\pi \xi)^{-1}(\lambda)\right|=2\right\}$ is a finite set.

We say that the image $D=\pi \xi(G)$ is a diagram of $G$.

Lemma 4.2. Let $G$ be as above and let $\xi: G \hookrightarrow \mathbb{R}^{3}$ be an embedding. For any projection $\pi: \mathbb{R}^{3} \rightarrow \mathbb{R}^{2}$, there is an embedding $\xi^{\prime}: G \hookrightarrow \mathbb{R}^{3}$ equivalent to $\xi$ such that $D=\pi \xi^{\prime}(G)$ is a diagram of $G$. 

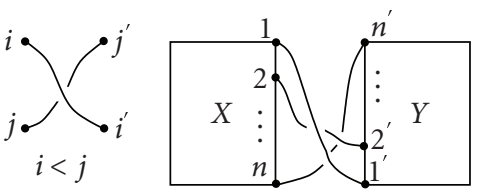

$D$

(a)
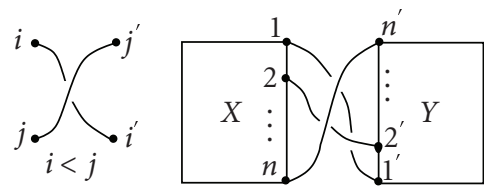

$D$

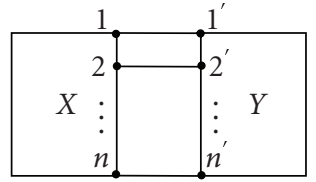

$D^{+}$

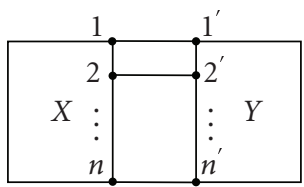

$D^{-}$

(b)

Figure 4.1

We say that $G$ is planar if there is an embedding $\xi^{\prime}: G \hookrightarrow \mathbb{R}^{2}$, that is, $\xi^{\prime}(e)$ are differentiable curves for edges $e$ of $G$ and $\xi^{\prime}(e), \xi^{\prime}\left(e^{\prime}\right)$ may only intersect in $\xi(x)$ if $x$ is a common vertex of $e$ and $e^{\prime}$.

4.3. Consider a hexagonal system $G$ and a diagram of $G$ of the form shown in Figure 4.1. In (a), we say that $D$ has a positive crossing and we define a diagram $D^{+}$which undoes the crossing. Similarly, in (b), we say that $D$ has a negative crossing and we define a diagram $D^{-}$which undoes the crossing. In general, we write $\bar{D}=D^{+}$or $D^{-}$in the above situations.

We say that $G$ is quasiplanar if there is a diagram $D=\operatorname{Im}\left(\pi \xi: G \rightarrow \mathbb{R}^{2}\right)$ and a finite sequence $D=D_{0}, D_{1}=\bar{D}_{0}, D_{2}=\bar{D}_{1}, \ldots, D_{s}=\bar{D}_{s-1}$ such that $D_{s}$ is planar.

Proposition 4.3. Let $G$ be a finite connected hexagonal system with boundary. If $G$ is simply connected, then $G$ is quasiplanar.

Proof. By induction on $h(G)$. If $h(G)=0$ and $\pi_{1}(G)=0$, then $G$ is a tree graph. Clearly $G$ is quasiplanar.

Assume $h(G) \geq 1$ and define $G^{\prime}$ as in (2.4) with $h\left(G^{\prime}\right)=h(G)-1$. By induction hypothesis, for the restriction $D^{\prime}=\pi \xi\left(G^{\prime}\right)$ of a diagram $D=\operatorname{Im}\left(\pi \xi: G \rightarrow \mathbb{R}^{2}\right)$, there is a sequence $D^{\prime}=D_{0}^{\prime}, D_{1}^{\prime}=\bar{D}_{0}^{\prime}, \ldots, D_{s}^{\prime}=\bar{D}_{s-1}^{\prime}$ such that $D_{s}^{\prime}$ is planar. We may add a hexagon at each step to get diagrams $D=D_{0}, D_{1}=\bar{D}_{0}, \ldots, D_{s}=\bar{D}_{s-1}$ such that $D_{i}^{\prime}$ is obtained from $D_{i}$ by deleting some edges of a hexagon.

Then either $D_{s}$ is planar or the situation in Figure 4.2 occurs (the crossing might be the opposite and observe that $e_{2}$ only belongs to a hexagon $h$ because $d\left(x_{2}\right) \leq 3$ and hexagons have at most one common edge). Hence $\bar{D}_{s}$ is planar, showing that $G$ is quasi-planar.

4.4. Remarks. (a) The crossings appearing in the covering defined in example (1.8) are of the type described at the above proof. 
14 The fundamental group of hexagonal systems in 3-space

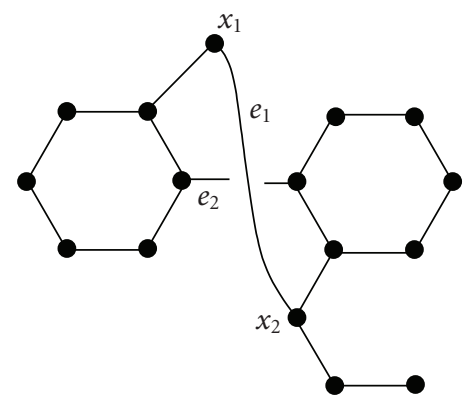

Figure 4.2

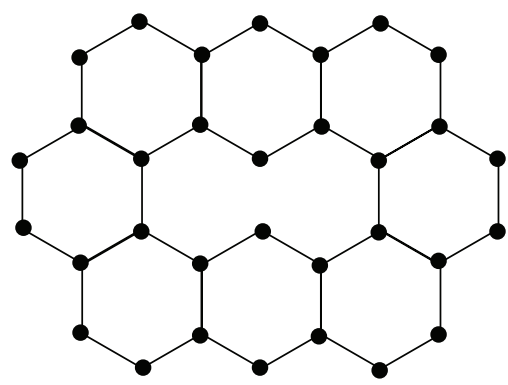

Figure 4.3

(b) The converse of the proposition does not hold as shown in Figure 4.3. $G$ is a planar hexagonal system with

$$
1=\operatorname{rk}(G), \quad m(G)-h(G)-n(G)=40-8-32=0 .
$$

\section{References}

[1] K. Bongartz and P. Gabriel, Covering spaces in representation-theory, Inventiones Mathematicae 65 (1982), no. 3, 331-378.

[2] D. M. Cvetković, M. Doob, and H. Sachs, Spectra of Graphs: Theory and Application, Pure and Applied Mathematics, vol. 87, Academic Press, New York, 1980.

[3] C. O. Dietrich-Buchecker and J.-P. Sauvage, A synthetic molecular Trefoil knot, Angewandte Chemie International Edition in English 28 (1989), no. 2, 189-192.

[4] E. Flapan, When Topology Meets Chemistry. A Topological Look at Molecular Chirality, Outlooks, Cambridge University Press, Cambridge; Mathematical Association of America, Washington, DC, 2000.

[5] P. Gabriel, The universal cover of a representation-finite algebra, Representations of Algebras (Puebla, 1980), Lecture Notes in Math., vol. 903, Springer, Berlin, 1981, pp. 68-105.

[6] J. L. Gross and T. W. Tucker, Topological Graph Theory, Wiley-Interscience Series in Discrete Mathematics and Optimization, John Wiley \& Sons, New York, 1987.

[7] I. Gutman and O. E. Polansky, Mathematical Concepts in Organic Chemistry, Springer, Berlin, 1986. 
[8] P. J. F. Harris, Carbon Nanotubes and Related Structures: New Materials for the Twenty-First Century, Cambridge University Press, Cambridge, 1999.

[9] R. Martínez-Villa and J. A. de la Peña, The universal cover of a quiver with relations, Journal of Pure and Applied Algebra 30 (1983), no. 3, 277-292.

[10] W. S. Massey, Algebraic Topology: An Introduction, Harcourt, Brace \& World, New York, 1967.

[11] Ch. Riedtmann, Algebren, Darstellungsköcher, Überlagerungen und zurück, Commentarii Mathematici Helvetici 55 (1980), no. 2, 199-224.

[12] D. M. Walba, R. M. Richards, and R. Curtis Haltiwanger, Total synthesis of the first molecular Möbius Band, Journal of the American Chemical Society 104 (1982), no. 11, 3219-3221.

[13] E. Wasserman, Chemical topology, Scientific American 207 (1962), no. 5, 94-100.

J. A. De La Peña: Instituto de Matemáticas, Universidad Nacional Autonoma de Mexico,

Cd. Universitaria, México 04510 DF, Mexico

E-mail address: jap@matem.unam.mx

L. Mendoza: Departamento de Matemáticas, Facultad de Ciencias, Universidad de los Andes, Mérida 5101, Venezuela

E-mail address: lmendoza@ula.ve 


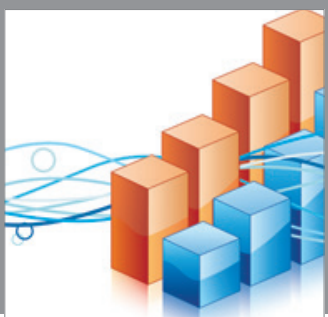

Advances in

Operations Research

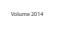

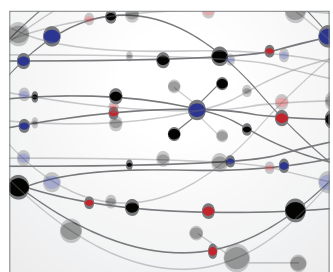

\section{The Scientific} World Journal
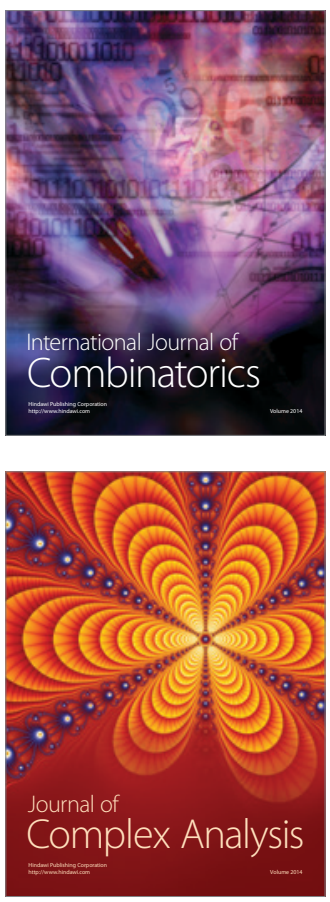

International Journal of

Mathematics and

Mathematical

Sciences
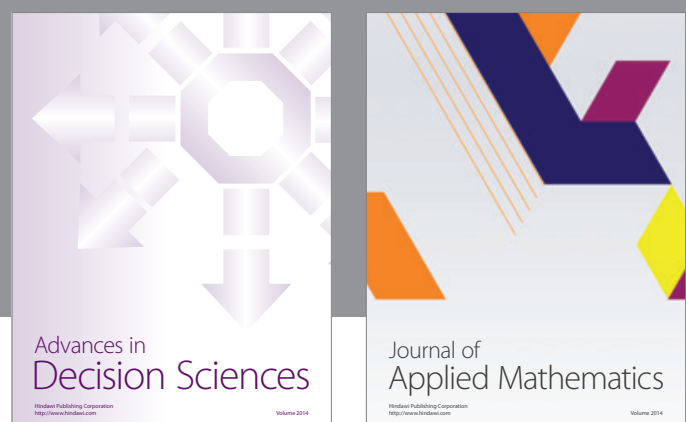

Journal of

Applied Mathematics
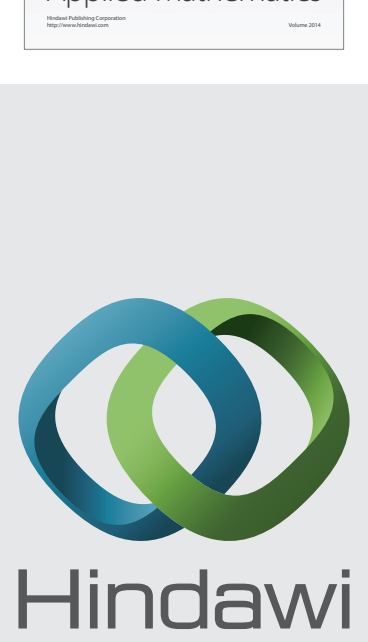

Submit your manuscripts at http://www.hindawi.com
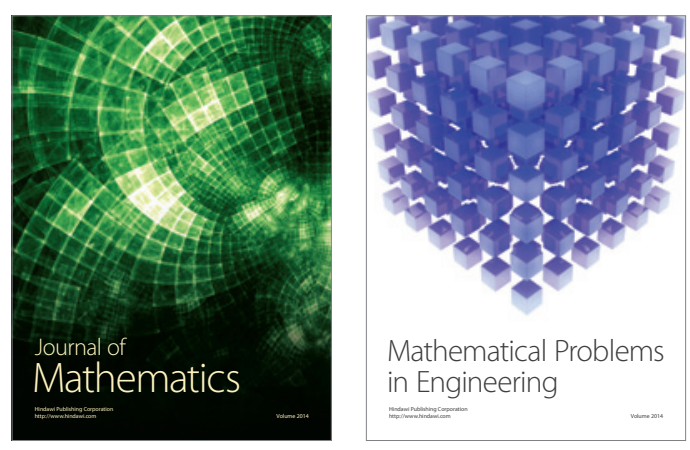

Mathematical Problems in Engineering
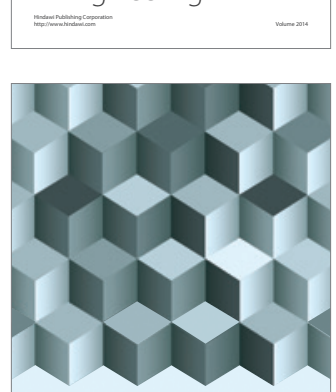

Journal of

Function Spaces
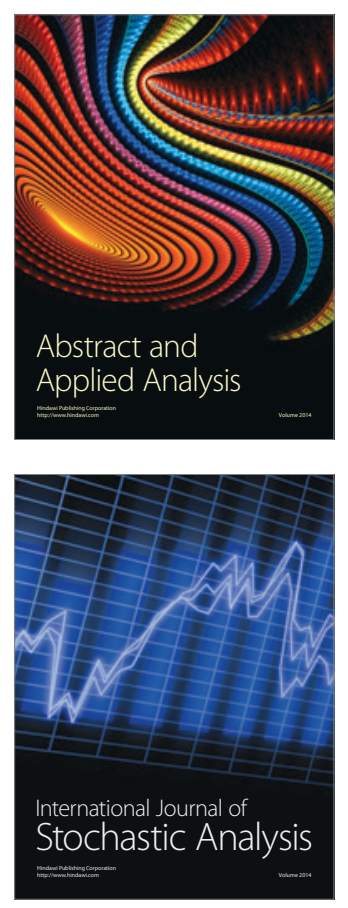

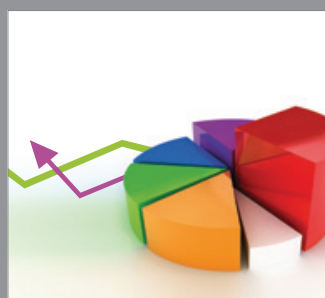

ournal of

Probability and Statistics

Promensencen
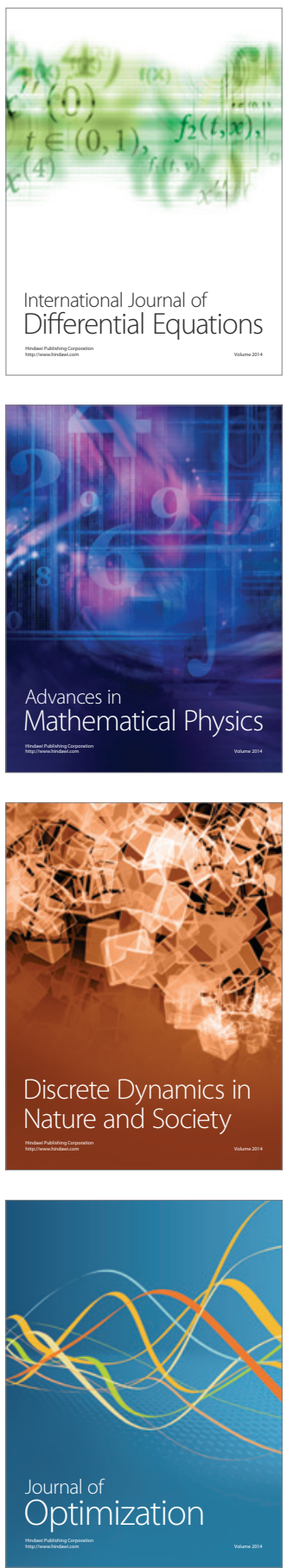